\title{
On the mutual noncompatibility of homogeneous analytic non-power means
}

\author{
MARCIN E. KuCZMA
}

Homogeneous symmetric means $\mu$ and $\tilde{\mu}$, defined on $\mathbb{R}_{+}^{n}$ and $\mathbb{R}_{+}^{n+1}$, respectively, are called compatible if the value of $\tilde{\mu}$ remains unchanged upon replacing $n$ of its arguments by their $\mu$-mean. Power means (of a common exponent) are a model example, which turns out to be unique, given analyticity of at least one of the two means considered. This is proved by fixing all but one argument in both $\mu$ and $\tilde{\mu}$, which leads to a functional equation with two unknown functions, involving their mutual superpositions. The equation is solved in the class of analytic functions by comparing the power series coefficients. 